\title{
$\begin{array}{ll}\text { Research Square } & \begin{array}{l}\text { Preprints are preliminary reports that have not undergone peer review. } \\ \text { They should not be considered conclusive, used to inform clinical practice, } \\ \text { or referenced by the media as validated information. }\end{array}\end{array}$
}

\section{Multiplex Lateral Flow Immunochromatographic Assay Is an Effective Method to Detect Carbapenemases Without Risk of OXA-48-like Cross Reactivity}

\section{Hadas Kon}

National Institute for Antibiotic Resistance and Infection Control, Ministry of Health

Shirin Abramov

National Institute for Antibioic Resistance and Infection Control, Ministry of Health

Sammy Frenk

National Institute for Antibiotic Resistance and Infection Control, Ministry of Health

David Schwartz

National Institute for Antibiotic Resistance and Infection Control, Ministry of Health

Ohad Shalom

Clinical Microbiology Laboratory, Tel-Aviv Sourasky Medical Center, Tel-Aviv

\section{Amos Adler}

Clinical Microbiology Laboratory, Tel-Aviv Sourasky Medical Center, Tel-Aviv; Sackler Faculty of Medicine, Tel-Aviv University, Israel

Yehuda Carmeli

National Institute for Antibiotic Resistance and Infection Control, Ministry of Health

Jonathan Lellouche ( $\square$ jonathanl@tlvmc.gov.il)

Sanz Medical Center: Laniado Hospital

\section{Research}

Keywords: NG-Test ${ }^{\circledR}$ CARBA 5 assay, cross reactivity, Enterobacterales, carbapenemase, OXA-48-like, carbapenem resistant

Posted Date: January 6th, 2021

DOI: https://doi.org/10.21203/rs.3.rs-138994/v1

License: (ㅇ (i) This work is licensed under a Creative Commons Attribution 4.0 International License. Read Full License 


\section{Abstract}

Background: It is essential to detect carriers of carbapenemase-producing Enterobacterales in order to implement infection control measures. The objectives of this study was to evaluate the NG-Test ${ }^{\circledR}$ CARBA 5 (CARBA 5) assay for detection of five carbapenemases and to assess the cross reactivity of other OXA-type carbapenemases with the OXA-48-like specific antibodies.

Methods: A total of 197 Enterobacterales isolates were tested. To evaluate the cross reactivity, 73 carbapenem-resistant $A$. baumannii, harboring OXA-type variants, were tested. Polymerase chain reaction (PCR) served as gold standard for carbapenemase identification.

Results: Excellent agreement was found between PCR and CARBA 5, for all but one isolate. The single false positive result (a bla $a_{\text {SME }}$ positive $S$. marcescens isolate) was incorrectly positive for bla $a_{\text {OXA-48 }}$ by CARBA 5 . No cross reactivity was observed. The sensitivity and specificity were $100.0 \%$ and $98.0 \%$, respectively.

Conclusions: The CARBA 5 assay is highly sensitive and specific and is recommended as a tool for the detection of five carbapenemases.

\section{Introduction}

Enterobacterales are a common cause of both community-acquired and hospital-acquired infections [1, 2]. These bacteria can acquire genes encoding multiple antibiotic resistance mechanisms, including extended-spectrum $\beta$-lactamases (ESBLs), AmpCs, and carbapenemases [3]. The global rise of carbapenemase-producing Enterobacterales (CPE) presents an increasing threat to healthcare delivery and patient safety [4].

Rapid detection of patients colonized or infected by CPE is necessary in order to provide a fast and correct treatment protocol. The NG-Test ${ }^{\circledR}$ CARBA 5 (CARBA 5) is a lateral flow immunoassay for carbapenemase detection, approved by the U.S Food and Drug Administration as of October 2019. CARBA 5 enables the detection of the five most prevalent carbapenemase enzymes (KPC, OXA48-like, VIM, IMP, and NDM) through the use of specific antibodies [5].

Because lateral flow immunoassays are based on antigen-antibody interaction, there is a risk of cross reactivity [6]. In the CARBA 5 assay, cross reactivity may occur if an isolate harboring an OXA-type variant reacts incorrectly with the OXA-48-like target, leading to a false positive result. Several studies have evaluated the CARBA 5 assay and found high sensitivity and specificity rates [7-15], yet only two of these studies evaluated the cross reactivity of other OXA-type carbapenemases with the OXA-48-like specific antibodies $[9,14]$. Here, we aimed to evaluate the CARBA 5 assay for detection of carbapenemases and to assess its specificity towards other OXA-type carbapenemases.

\section{Materials And Methods}

Isolate selection. The isolates in our sample were selected in order to represent different years and hospitals from the collection of Enterobacterales isolates that were sent to the National Institute for Antibiotic Resistance \& Infection Control in the years 20012017. An initial screening for carbapenemase identification was performed using polymerase chain reaction (PCR). We used a multiplex assay to detect $b / a_{\mathrm{KPC}}, b / a_{\mathrm{OXA}-48-\mathrm{like}}, b / a_{\mathrm{NDM}}, b / a_{\mathrm{IMP}}, b / a_{\mathrm{VIM}}$, and $b / a_{\mathrm{IMI}}[16]$ and a simplex PCR to detect $b / a_{\mathrm{SME}}[17]$. The PCR results served as the gold standard to which the CARBA-5 assay was compared. Carbapenemase activity was assessed using the qualitative colorimetric $\beta$ CARBA test (Bio-Rad, Marnes-la-Coquette, France) according to the manufacturer's instructions.

The sample consisted of 197 unrelated, non-duplicate isolates from various sources (sputum $n=51$, blood $n=74$, urine $n=48$, rectal $\mathrm{n}=20$, other $\mathrm{n}=4)$. There were $19(19 / 197,9.6 \%)$ carbapenem-susceptible Enterobacterales isolates (CSE; meropenem MIC < $4 \mu \mathrm{g} / \mathrm{ml}$ ) and $178(178 / 197,90.4 \%)$ carbapenem-resistant Enterobacterales (CRE; meropenem MIC $\geq 8 \mu \mathrm{g} / \mathrm{ml})$. Of the $178 \mathrm{CRE}$

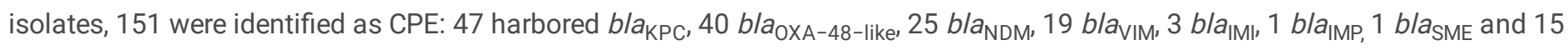
isolates harbored two or three carbapenemases. The other $27 \mathrm{CRE}$ isolates (11 K. pneumoniae, 7 E. coli, 4 Enterobacter spp., 1 K. oxytoca, 1 Proteus spp., 1 E. aerogenes and 2 Providencia spp.) had meropenem MIC values of $\geq 8 \mu \mathrm{g} / \mathrm{ml}$ but no carbapenemase activity and none of the carbapenemases genes screened for in this study. They were classified as non-carbapenemase-producing CRE (Non-CP CRE). 
All isolates were stored at $-80^{\circ} \mathrm{C}$, sub-cultured aerobically at $35 \pm 2{ }^{\circ} \mathrm{C}$ and transferred twice prior to testing.

OXA-48-like target cross reactivity evaluation. To evaluate the cross reactivity with the OXA-48-like target, we tested 73 carbapenemresistant $A$. baumannii (CRAB) isolates (meropenem $\mathrm{MIC} \geq 8 \mu \mathrm{g} / \mathrm{ml}$ ), all of them Ambler class $\mathrm{D} \beta$-lactamases producers. $A$. baumannii isolates were identified by Vitek MS (BioMe'rieux, Marcy-l'Étoile, France). Isolates were tested by PCR for the bla $a_{0 X A-24}$ bla $a_{\text {OXA-23 }}$ and intrinsic bla OXA-51-LIKE genes, as described previously [18]. PCR products were sequenced and OXA-type variants were determined using DNAMAN® software version 7.0 (Lynnon Corporation, Pointe-Claire, Quebec) and the Basic Local Alignment Search Tool (BLAST, http://blast.ncbi.nlm.nih.gov). The CRAB isolates harbored bla OXA- $23(n=6), b / a_{\text {OXA- } 65}(n=6), b / a_{0 X A} 66(n=$ 11), bla OXA- $69(\mathrm{n}=2)$, bla OXA-70 $(\mathrm{n}=4)$, bla OXA-71 $(\mathrm{n}=11)$, bla OXA- $248(\mathrm{n}=8)$ and various OXA-type combinations $(\mathrm{n}=25)$.

The CRAB isolates were stored at $-80^{\circ} \mathrm{C}$, sub-cultured aerobically at $35 \pm 2{ }^{\circ} \mathrm{C}$ and transferred twice prior to testing.

NG-Test CARBA 5 assay. The CARBA 5 assay (NG Biotech, Guipry, France) is based on the reaction of carbapenemases with labelled anti-carbapenemase monoclonal antibodies. The assay was performed using fresh colonies grown on CHROMagar ${ }^{\text {TM }}$ mSuperCARBA ${ }^{\text {Tm }}$ (Hy Laboratories, Rehovot, Israel) or Mueller Hinton agar (Hy Laboratories), as recommended in the CARBA 5 instruction manual. One colony was suspending in five drops $(150 \mu \mathrm{L})$ of extraction buffer. The bacterial suspension was homogenized by vortex and $100 \mu \mathrm{l}$ was loaded into a nitrocellulose membrane. The suspension migrated through the membrane due to capillary force, and interacted with the corresponding anti-carbapenemase monoclonal antibodies immobilized on the membrane. Results were read by researchers blinded to the PCR results, to ensure unbiased interpretation, following 15 min of incubation time at room temperature. Results were considered positive if a red line appeared on the control region and on one or more of the test regions (KPC, OXA, VIM, IMP or NDM), indicating that the isolate carried one or more carbapenemases. Results were considered negative if a red line appeared only on the control line, indicating that the isolate did not carry any of the five carbapenemases.

Discrepancies between the CARBA 5 results and the PCR results were further investigated by genome sequencing.

Genome sequencing. Isolates requiring investigation by genome sequencing were grown overnight on Brain Heart Infusion broth (Hy Laboratories) and DNA was extracted using the MagAttract HMW DNA Kit (Qiagen, Hilden, Germany). Purified DNA was sequenced using the Rapid Barcoding Sequencing (Ref. No. SQK-RBK004, Oxford Nanopore Technologies, Oxford, UK), on a MinION sequencing device (Oxford Nanopore Technologies). FastQ files were subjected to antibiotic resistance gene search using ResFinder K-mer alignment web interface [19]. Genome sequence was submitted to GenBank under BioProject no. SAMN17121013.

Statistical analysis. Using PCR as the gold standard, we calculated the sensitivity and specificity of CARBA 5. We calculated 95\% confidence intervals (Cl) for each of these measures using VassarStats (http://vassarstats.net/prop1.html). If CARBA 5 detected the presence of a carbapenemase, but it differed from the carbapenemase detected by PCR, we considered the result a false positive (FP).

\section{Results}

Confirmation of the initial screening for carbapenemase identification results was performed by PCR. Details of the Enterobacterales isolates are shown in Table 1. The $\beta$ CARBA test confirmed the expression of carbapenemases for $148 / 151$ CPE isolates; it did not detect carbapenemase activity in the three isolates harboring $b / a_{\mathrm{IM}}$, a gene for which some variants are not detectable by this test [20]. 
Table 1

Details of Enterobacterales isolates characterized by PCR

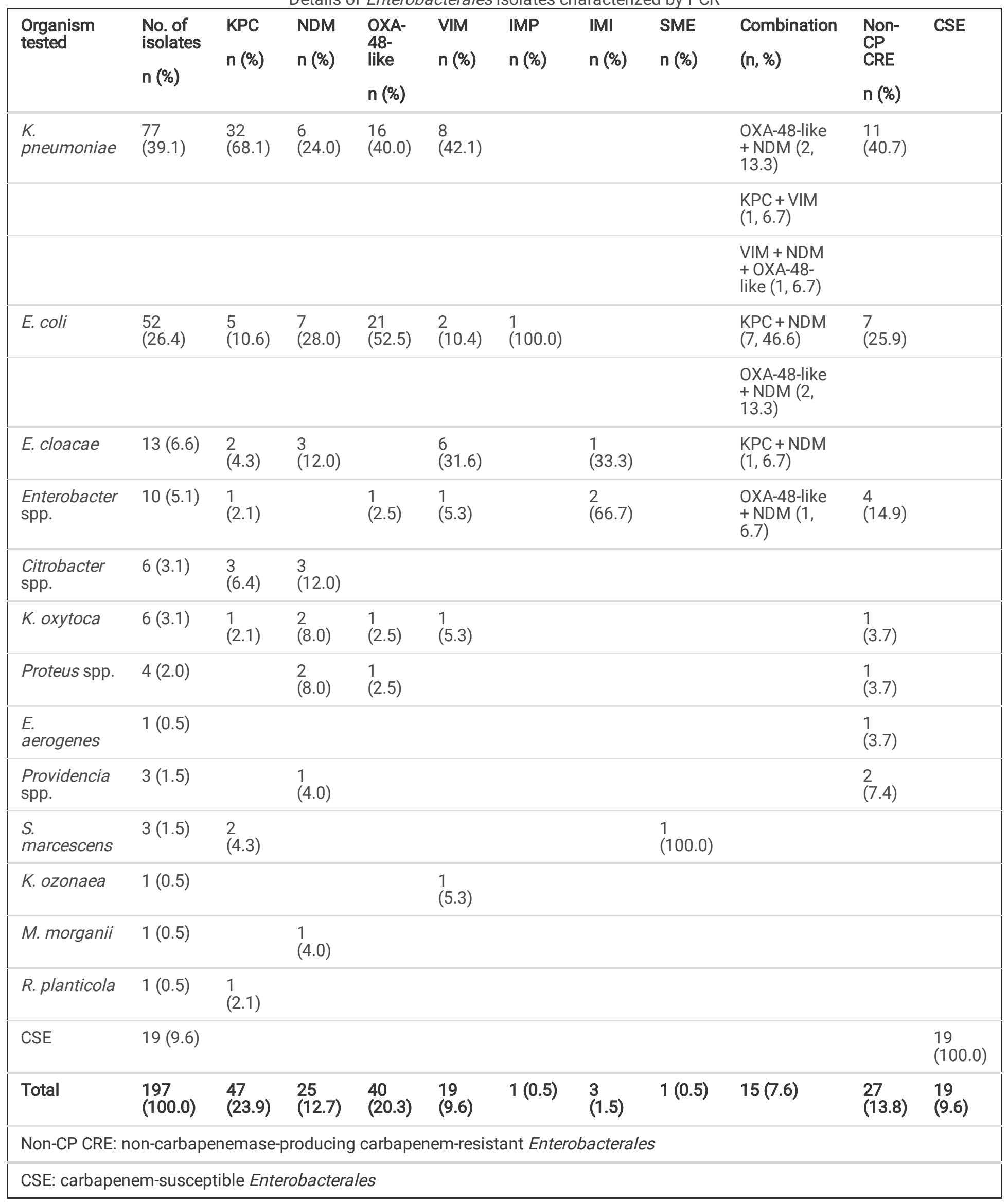

Page $4 / 9$ 
The CARBA 5 assay accurately detected carbapenemase production in all 147 CPE isolates expressing a carbapenemase that the assay is designed to detect (KPC, OXA-48-like, VIM, IMP, and NDM); including isolates that were double or triple carbapenemase producers. Although results were read after $15 \mathrm{~min}$, as per the manufacturer's instructions, in most cases a positive result was obtained after 3-6 min. No false negative (FN) results were observed.

The CARBA 5 assay accurately identified as non-carbapenemase producers all 19 CSE and all 27 non-CP CRE. Additionally, the three CPE isolates carrying $b / a_{\mid M I}$ were identified as negative, as IMI is not targeted by CARBA 5. There was one discrepancy between PCR and CARBA 5 results: a $S$. marcescens isolate expressing a bla $a_{\text {SME }}$ gene according to PCR and b/a $a_{0 X A-48}$ according to CARBA 5. Genome sequencing confirmed the presence of $b / a_{\text {SME }}$ and the absence of a bla $a_{\text {OXA-48 }}$ gene or one of its variants. We classified this error as a FP result. For this isolate, the red line on the CARBA 5 indicating a positive OXA-48 result, appeared only after 14 minutes and was faintly visible.

Details of the 73 A. baumannii isolates harboring OXA-type variants are presented in Table 2. The isolates harbored one, two or three OXA-type variants simultaneously (OXA-23, -24/-40, -65, -66, -69, -70, -71, -82, -90, and - 248). All 73 CRAB isolates harboring OXAvariants were correctly tested negative.

Table 2

Details of $A$. baumannii isolates harboring OXA-type variants

\begin{tabular}{|c|c|c|c|c|c|c|c|c|}
\hline $\begin{array}{l}\text { OXA- } \\
23 \\
\text { n (\%) }\end{array}$ & $\begin{array}{l}\text { OXA- } \\
65 \\
\text { n (\%) }\end{array}$ & $\begin{array}{l}\text { OXA-66 } \\
n(\%)\end{array}$ & $\begin{array}{l}\text { OXA- } \\
69 \\
n(\%)\end{array}$ & $\begin{array}{l}\text { OXA- } \\
70 \\
\text { n (\%) }\end{array}$ & $\begin{array}{l}\text { OXA-71 } \\
\text { n (\%) }\end{array}$ & $\begin{array}{l}\text { OXA-248 } \\
\text { n (\%) }\end{array}$ & $\begin{array}{l}\text { Combinations } \\
(n, \%)\end{array}$ & $\begin{array}{l}\text { Total } \\
\text { n (\%) }\end{array}$ \\
\hline \multirow[t]{6}{*}{$6(8.2)$} & \multirow[t]{6}{*}{$6(8.2)$} & \multirow{6}{*}{$\begin{array}{l}11 \\
(15.1)\end{array}$} & \multirow[t]{6}{*}{$2(2.7)$} & \multirow[t]{6}{*}{$4(5.4)$} & \multirow[t]{6}{*}{$11(15.1)$} & \multirow[t]{6}{*}{$8(11.0)$} & OXA-23 + OXA-66 $(8,11.0)$ & \multirow[t]{6}{*}{$73(100.0)$} \\
\hline & & & & & & & OXA-71 + OXA-23 $(11,15.1)$ & \\
\hline & & & & & & & OXA-23 + OXA-82 $(2,2.7)$ & \\
\hline & & & & & & & OXA-65 + OXA-71 + OXA-23 $(1,1.4)$ & \\
\hline & & & & & & & OXA-24 + OXA-71 + OXA-23 $(2,2.7)$ & \\
\hline & & & & & & & OXA-24 + OXA-90 $(1,1.4)$ & \\
\hline
\end{tabular}

In our sample, CARBA 5 had 100.0\% sensitivity (95\% Cl: $97.4 \%-100.0 \%)$ and $98.0 \%$ specificity (95\% Cl: 89.5\%-99.6\%).

\section{Discussion}

Identification of carbapenemase genes can be done either by molecular tests, which detect the presence of a resistant carbapenemase gene, or by phenotypic tests, which detect the in vitro activity of carbapenemase enzymes [21, 22]. Molecular techniques, such as PCR, have several advantages, including accurate results and high sensitivity. However, molecular techniques cannot detect new or mutated genes and the implementation of these techniques requires skilled technicians [23]. Phenotypic tests, which are based on hydrolysis of a carbapenem (most often imipenem and meropenem), detect direct or indirect degradation products. Phenotypic tests include the Carba NP test [24], the modified carbapenem inactivation method (mCIM), the EDTA-modified carbapenem inactivation method (eCIM) and the rapid carbapenem inactivation method ( $\mathrm{rCIM})[25,26]$. Although the Carba NP test is recommended by the Clinical and Laboratory Standards Institute (CLSI) [27], a significant drawback of this test is the long preparation time required. Commercial derivatives of the Carba NP test are available and have simpler protocols, such as the BlueCarba test [28]. The $\mathrm{mCIM}$ attain a high sensitivity and specificity, but the long turnaround time required (18 to 24 hours) is a major limitation. Both the hydrolysis-based assays and the $\mathrm{mCIM}$ have limited ability to determine the exact carbapenemase enzyme, which might have important epidemiological and clinical implications.

In this study, we evaluated the performance of the CARBA 5 assay, a lateral flow immunoassay which is a simple, rapid and low-cost tool for carbapenemase detection. We found excellent agreement between the carbapenemase genes detected by PCR and the results of CARBA 5 , for all but one isolate. The single FP result, obtained on a bla $a_{\text {SME }}$ positive $S$. marcescens isolate, was incorrectly positive for $b / a_{\mathrm{OXA}-48}$ by the CARBA 5 assay. 
Previous studies evaluating the CARBA 5 assay found a sensitivity ranging from $88-100 \%$ and a specificity varying between $95-$ $100 \%[7-15,29]$. Three of these studies demonstrated $100 \%$ agreement $[7,12,15]$. Five evaluations reported FN errors obtained by isolates harboring IMP, NDM, VIM or OXA-48 [8-11, 13, 29]; whereas FP errors were reported in only three studies, attained from isolates carrying VIM or OXA-48-like genes $[8,10,14]$. In contrast to these evaluations, our study did not result in any FN errors, and obtained a single FP error.

The advantages of the CARBA 5 assay are numerous. CARBA 5 requires minimal preparation time and results are obtained within $15 \mathrm{~min}$. The assay can be easily implemented in clinical microbiology laboratories and does not require external equipment or maintenance expenses. CARBA 5 enables detection of strains which express two or three carbapenemases simultaneously in a single test assay. It should be noted that the CARBA 5 assay cannot detect the IMI gene, therefore $E$. cloacae isolates with reduced susceptibility to meropenem need to be tested by other methods.

An alternative lateral flow immunoassay, RESIST-4 O.K.N.V. (Coris BioConcept, Gembloux, Belgium), is available for the detection of up to four carbapenemases. Several studies evaluating this test reported a specificity of $100 \%$ and a sensitivity ranging between

$84-100 \%$. Most errors were caused by isolates harboring $b / a_{\text {NDM }}(83-95 \%$ sensitivity for NDM) [11, 30-35]. The advantage of CARBA 5 is that it can detect an additional carbapenemase that RESIST-4 O.K.N.V. cannot: bla $\left.\right|_{\text {IMP. }}$

Two prior studies evaluated the CARBA 5 for cross reactivity with the OXA-48-like target. Potron et al. (2019) evaluated cross reactivity on a small sample of 19 Acinetobacter spp., each harboring a single OXA-type variant (OXA-23, -24/-40, or -58) [9]. Boutal et al. (2018) tested cross reactivity using enterobacterial isolates that harbored OXA-1, -2, -9 or -10 [14]. Both of those studies, like our own, found no cross reactivity, demonstrating the low likelihood of an FP result generated by Acinetobacter in a heterogeneous culture.

In conclusion, the CARBA 5 assay is highly sensitive and specific, rapid, and easy to implement in routine workflow. It is an accurate tool for the detection of five main carbapenemases for diagnostic purposes, infection control and outbreak prevention.

\section{Abbreviations}

CARBA 5: NG-Test ${ }^{\circledR}$ CARBA 5

PCR: Polymerase chain reaction

ESBLs: extended-spectrum $\beta$-lactamases

CPE: carbapenemase-producing Enterobacterales

CSE: carbapenem-susceptible Enterobacterales isolates

Non-CP CRE: non-carbapenemase-producing CRE

CRAB: carbapenem-resistant A. baumannii

Cl: confidence intervals

FP: false positive

FN: false negative

MCIM: modified carbapenem inactivation method

ECIM: EDTA-modified carbapenem inactivation method

RCIM: rapid carbapenem inactivation method

CLSI: Clinical and Laboratory Standards Institute 


\section{Declarations}

Ethics declarations

Ethics approval and consent to participate

The study was performed as part of the routine work of the National Center for Antibiotic Resistance and Infection Control, Ministry of Health. No ethics approval or participant consent was required for this study using de-identified isolates. No human subjects were involved in this study.

\section{Consent for publication}

Not applicable.

\section{Competing interests}

The authors declare that they have no competing interests.

Availability of data and materials

Not applicable.

\section{Funding}

This research did not receive any specific grant from funding agencies in the public, commercial, or not-for-profit sectors.

\section{Authors' contributions}

Writing - Original Draft. H.K.; Writing - Review \& Editing. J.L., O.S, A.A and Y.C.; Conceptualization: J.L., and Y.C.; Investigation: S.A., O.S; Methodology. J.L., D.S., and Y.C.; Formal Analysis: H.K.; Bioinformatics analysis: S.F; Supervision: J.L.; Funding Acquisition: Y.C.

\section{Acknowledgments}

The authors would like to acknowledge Dr. Elizabeth Temkin who provided critical review of the manuscript.

\section{References}

1. Peleg AY, Hooper DC. Hospital-acquired infections due to gram-negative bacteria. N. Engl. J. Med. 2010;doi: 10.1056/NEJMra0904124

2. Tang HJ, Hsieh CF, Chang PC, Chen JJ, Lin YH, Lai CC, et al. Clinical significance of community-and healthcare-acquired carbapenem-resistant Enterobacteriaceae isolates. PLoS One 2016;doi: 10.1371/journal.pone.0151897

3. Codjoe FS, Donkor ES. medical sciences Review Carbapenem Resistance: A Review. Med. Sci. 2017; doi: $10.3390 /$ medsci6010001

4. Logan LK, Weinstein RA. The epidemiology of carbapenem-resistant Enterobacteriaceae: The impact and evolution of a global menace. J. Infect. Dis. 2017;doi: 10.1093/infdis/jiw282

5. Tamma PD, Simner PJ. Phenotypic detection of carbapenemase-producing organisms from clinical isolates. J. Clin. Microbiol. 2018;doi: 10.1128/JCM.01140-18

6. Wild D, Kodak E. The Immunoassay Handbook. 2013. doi: 10.1016/C2010-0-66244-4

7. Stephen Jenkins, Nathan A Ledeboer, Lars F Westblade, C A Burnham, et al. Evaluation of NG-Test CARBA 5 for rapid phenotypic detection and differentiation of five common carbapenemase families: Results of a multicenter clinical evaluation. J Clin Microbiol. 2020;

8. Hopkins KL, Meunier D, Naas T, Volland H, Woodford N. Evaluation of the NG-Test CARBA 5 multiplex immunochromatographic assay for the detection of KPC, OXA-48-like, NDM, VIM and IMP carbapenemases. J. Antimicrob. Chemother. 2018;doi:

10.1093/jac/dky342 
9. Potron A, Fournier D, Emeraud C, Triponney P, Plésiat P, Naas T, et al. Evaluation of the immunochromatographic NG-test CARbA 5 for rapid identification of carbapenemase in nonfermenters. Antimicrob. Agents Chemother. 2019;doi: 10.1128/AAC.00968-19

10. Takissian J, Bonnin RA, Naas T, Dortet L. NG-test carba 5 for rapid detection of carbapenemase-producing Enterobacterales from positive blood cultures. Antimicrob. Agents Chemother. 2019;doi: 10.1128/AAC.00011-19

11. Baeza LL, Pfennigwerth N, Greissl C, Göttig S, Saleh A, Stelzer Y, et al. Comparison of five methods for detection of carbapenemases in Enterobacterales with proposal of a new algorithm. Clin. Microbiol. Infect. 2019;doi:

10.1016/j.cmi.2019.03.003

12. Kieffer N, Poirel L, Nordmann P. Rapid immunochromatography-based detection of carbapenemase producers. Infection 2019;doi: 10.1007/s15010-019-01326-1

13. Davison BD. Comparison of the NG biotech NG-test CARBA 5 and CORIS bioconcept RESIST-4 O.K.N.V. immunochromatographic lateral flow assays for the detection of carbapenemase enzymes in Enterobacterales. New Zeal. J. Med. Lab. Sci. 2019;

14. Boutal H, Vogel A, Bernabeu S, Devilliers $K$, Creton E, Cotellon G, et al. A multiplex lateral flow immunoassay for the rapid identification of NDM-, KPC-, IMP- and VIM-type and OXA-48-like carbapenemase-producing Enterobacteriaceae. J. Antimicrob. Chemother. 2018;doi: 10.1093/jac/dkx521

15. Bodendoerfer E, Keller PM, Mancini S. Rapid identification of NDM-, KPC-, IMP-, VIM- And OXA-48-like carbapenemase-producing Enterobacteriales from blood cultures by a multiplex lateral flow immunoassay. J. Antimicrob. Chemother. 2019;doi: $10.1093 / j a c / d k z 056$

16. Poirel L, Walsh TR, Cuvillier V, Nordmann P. Multiplex PCR for detection of acquired carbapenemase genes. Diagn. Microbiol. Infect. Dis. 2011;doi: 10.1016/j.diagmicrobio.2010.12.002

17. Mlynarcik P, Roderova M, Kolar M. Primer evaluation for PCR and its application for detection of carbapenemases in Enterobacteriaceae. Jundishapur J. Microbiol. 2016;doi: 10.5812/jjm.29314

18. Hou C, Yang F. Drug-resistant gene of blaOXA-23, blaOXA-24, blaOXA-51 and blaOXA-58 in Acinetobacter baumannii. Int. J. Clin. Exp. Med. 2015;

19. Clausen PTLC, Aarestrup FM, Lund O. Rapid and precise alignment of raw reads against redundant databases with KMA. BMC Bioinformatics 2018; doi: 10.1186/s12859-018-2336-6

20. BIO-RAD. $\beta$ CARBA Test: Rapid detection of carbapenemase-producing Enterobacteriaceae strains. 2015;1-6. doi: http://www.bio-rad.com/webroot/web/pdf/inserts/CDG/en/68260_881159_EN.pdf

21. Főldes A, Bilca DV, Székely E. Phenotypic and molecular identification of carbapenemase-producing enterobacteriaceaechallenges in diagnosis and treatment. Rev. Rom. Med. Lab. 2018;doi: 10.2478/rrlm-2018-0018

22. Iovleva A, Doi Y. Carbapenem-resistant Enterobacteriaceae. Clin. Lab. Med. 2017;doi: 10.1016/j.cll.2017.01.005

23. Nordmann P, Gniadkowski M, Giske CG, Poirel L, Woodford N, Miriagou V, et al. Identification and screening of carbapenemaseproducing Enterobacteriaceae. Clin. Microbiol. Infect. 2012;doi: 10.1111/j.1469-0691.2012.03815.x

24. Nordmann P, Poirel L, Dortet L. Rapid detection of carbapenemase-producing Enterobacteriaceae. Emerg. Infect. Dis. 2012;doi: 10.3201/eid1809.120355

25. Li J, Li C, Cai X, Shi J, Feng L, Tang K, et al. Performance of modified carbapenem inactivation method and inhibitor-based combined disk test in the detection and distinguishing of carbapenemase producing Enterobacteriaceae. Ann. Transl. Med. 2019;doi: 10.21037/atm.2019.09.43

26. Muntean MM, Muntean AA, Gauthier L, Creton E, Cotellon G, Popa MI, et al. Evaluation of the rapid carbapenem inactivation method (rCIM): A phenotypic screening test for carbapenemase-producing Enterobacteriaceae. J. Antimicrob. Chemother. 2018;doi: 10.1093/jac/dkx519

27. Franklin R. Cockerill, III M, Jean B. Patel, PhD D. M100-S25 Performance standards for antimicrobial susceptibility testing; Twenty-fifth informational supplement. Clin. Lab. Stand. Inst. 2015;doi: 10.1186/1476-0711-9-23

28. Pires J, Novais A, Peixe L. Blue-Carba, an easy biochemical test for detection of diverse Carbapenemase producers directly from bacterial cultures. J. Clin. Microbiol. 2013;doi: 10.1128/JCM.01634-13

29. Chan WW, Campbell L, Doyle D, Pitout JD. Rapid detection of Enterobacterales that produce carbapenemases. Diagn. Microbiol. Infect. Dis. 2020;doi: 10.1016/j.diagmicrobio.2020.115120

Page $8 / 9$ 
30. MacDonald JW, Chibabhai V. Evaluation of the RESIST-4 O.K.N.V immunochromatographic lateral flow assay for the rapid detection of OXA-48, KPC, NDM and VIM carbapenemases from cultured isolates. Access Microbiol. 2019;doi:

10.1099/acmi.0.000031

31. Saleh A, Göttig S, Hamprecht AG. Multiplex immunochromatographic detection of OXA-48, KPC, and NDM carbapenemases: Impact of inoculum, antibiotics, and agar. J. Clin. Microbiol. 2018;doi: 10.1128/JCM.00050-18

32. Kolenda C, Benoit R, Carricajo A, Bonnet R, Dauwalder O, Laurenta F. Evaluation of the new multiplex immunochromatographic O.K.N.V. K-SeT assay for rapid detection of OXA-48-like, KPC, NDM, and VIM carbapenemases. J. Clin. Microbiol. 2018;doi: 10.1128/JCM.01247-18

33. Glupczynski Y, Jousset A, Evrard S, Bonnin RA, Huang TD, Dortet L, et al. Prospective evaluation of the OKN K-SeT assay, a new multiplex immunochromatographic test for the rapid detection of OXA-48-like, KPC and NDM carbapenemases. J. Antimicrob. Chemother. 2017; doi: 10.1093/jac/dkx089

34. GreissI C, Saleh A, Hamprecht A. Rapid detection of OXA-48-like, KPC, NDM, and VIM carbapenemases in Enterobacterales by a new multiplex immunochromatographic test. Eur. J. Clin. Microbiol. Infect. Dis. 2019;doi: 10.1007/s10096-018-3432-2

35. Wareham DW, Momin MHFA. Rapid detection of carbapenemases in Enterobacteriaceae: Evaluation of the resist-3 O.K.N. (OXA48, KPC, NDM) lateral flow multiplexed assay. J. Clin. Microbiol. 2017;doi: 10.1128/JCM.02471-16 